N. E. Piskunov, W. W. Weiss, D. F. Gray, eds.

\title{
Highlights of Stellar Modeling with PHOENIX
}

\author{
E. Baron ${ }^{1}$, P.H. Hauschildt ${ }^{2,3}$, F. Allard ${ }^{4}$, E.J. Lentz ${ }^{2}$, J. Aufdenberg ${ }^{5}$, \\ A. Schweitzer ${ }^{2,3}$, and T. Barman ${ }^{2,6}$
}

\begin{abstract}
We briefly describe the current version of the PHOENIX code. We then present some illustrative results from the modeling of Type Ia and Type II supernovae, hot stars, and irradiated giant planets. Good fits to observations can be obtained, when account is taken for spherically symmetric, line-blanketed, static or expanding atmospheres.
\end{abstract}

\section{Introduction}

PHOENIX (see Hauschildt \& Baron, 1999, and references therein) is a generalized, stellar model atmosphere code for treating both static and moving atmospheres. The goal of PHOENIX is to be both as general as possible so that essentially all astrophysical objects can be modeled with a single code, and to make as few approximations as possible. Approximations are inevitable (particularly in atomic data where laboratory values for most quantities are unknown); however, the agreement of synthetic spectra with observations across a broad class of astrophysical objects is a very good consistency check. We have modeled Planets/BDs (Barman et al., 2002; Allard et al., 2001; Schweitzer et al., 2001, 2002), Cool Stars (Hauschildt et al., 1999; Hauschildt et al., 1999), Hot Stars ( $\beta \mathrm{CMa}, \epsilon \mathrm{CMa}$, Deneb - Aufdenberg et al., 1998, 1999, 2002), $\alpha$-Lyra, Novae (Hauschildt et al., 1997b; Schwarz et al., 1997), and all types of superovae (SNe Ia, Ib/c, IIP, IIb Baron et al., 1995; Lentz et al., 2001a; Baron et al., 1999; Mitchell et al., 2002).

\section{The PHOENIX Code}

PHOENIX solves the radiative transfer problem by using the short-characteristic method (Olson et al., 1987; Olson \& Kunasz, 1987) to obtain the formal solu-

\footnotetext{
${ }^{1}$ Dept. of Physics and Astronomy, University of Oklahoma, 440 W. Brooks, Rm. 131, Norman, OK 73019 USA

${ }^{2}$ Dept. of Physics \& Astronomy and Center for Simulational Physics, University of Georgia, Athens, GA 30602-2451 USA

${ }^{3}$ Present Address: Hamburger Sternwarte, Gojenbergsweg 112, 21029 Hamburg, Germany

${ }^{4}$ CRAL, Ecole Normale Superieure, 46 Allee d'Italie, Lyon, 69364 France, Cedex 07

${ }^{5}$ Harvard-Smithsonian Center for Astrophysics, Mail Stop 15, 60 Garden Street Cambridge, MA 02138 USA

${ }^{6}$ Present Address: Dept. of Physics, Wichita State University, Wichita, KS 67260-0032 USA
} 
tion of the special relativistic, spherically symmetric radiative transfer equation (SSRTE) along its characteristic rays. The scattering problem is solved via the use of a band-diagonal approximation to the discretized $\Lambda$-operator (Hauschildt, 1992; Olson \& Kunasz, 1987; Hauschildt et al., 1994) as our choice of the approximate $\Lambda$-operator. This method can be implemented very efficiently to obtain an accurate solution of the SSRTE for continuum and line transfer problems using only modest amounts of computer resources.

We emphasize that PHOENIX solves the radiative transfer problem including a full treatment of special relativistic radiative transfer in spherical geometry for all lines and continua. In addition we enforce the generalized condition of radiative equilibrium in the Lagrangian frame, including all velocity terms and deposition of energy from radiative decay or from external irradiation.

We also include a full non-LTE treatment of most ions, using model atoms constructed from the data of Kurucz $(1993,1994 a, b)$ and/or from the CHIANTI database http://wwwsolar.nrl.navy.mil/chianti.html. The code uses Fortran-95 data structures to access the different databases and model atoms from either or both databases can be selected at execution time.

Absorption and emission is treated assuming complete redistribution and detailed depth-dependent profiles for the lines. Fluorescence effects are included in the NLTE treatment. The equation of state used includes up to 26 ionization stages of 40 elements as well as up to 206 molecules.

The atomic data is constructed from all relevant b-f and $\mathrm{f}-\mathrm{f}$ transitions (Mathisen, 1984; Verner \& Yakovlev, 1995; Seaton et al., 1994) as well as collisional rates obtained from laboratory measurements (where available), the Opacity Project, the Iron Project, Van Regemorter's formula (Van Regemorter, 1962), and the semi-empirical formula from Allen (1973).

In addition to NLTE lines, lines treated in LTE are selected dynamically from the $42 \times 10^{6}$ list of Kurucz (1993). A typical calculation includes about $2 \times 10^{6}$ lines that are treated as background opacity (Hauschildt \& Baron, 1999). Molecular opacities (about $7 \times 10^{8}$ lines) are discussed in Allard et al. (2001) and molecular NLTE is discussed in Schweitzer et al. (2000).

\section{The equation of radiative transfer}

The equation of transfer in spherical coordinates in the co-moving frame can be written (Mihalas \& Mihalas, 1984):

$$
e \frac{\partial I}{\partial r}+\frac{\partial}{\partial \mu}(f I)+g \frac{\partial}{\partial \lambda}(\lambda I)+h I=\eta-\chi I
$$

with

$$
\begin{aligned}
& e(r, \mu)=\gamma(\mu+\beta) \\
& f(r, \mu)=\gamma\left(1-\mu^{2}\right)\left[\frac{1+\beta \mu}{r}-\gamma^{2}(\mu+\beta) \frac{\partial \beta}{\partial r}\right] \\
& g(r, \mu)=\gamma\left[\frac{\beta\left(1-\mu^{2}\right)}{r}+\gamma^{2} \mu(\mu+\beta) \frac{\partial \beta}{\partial r}\right]
\end{aligned}
$$




$$
h(r, \mu)=\gamma\left[\frac{\beta\left(1-\mu^{2}\right)}{r}+\gamma^{2}\left(1+\mu^{2}+2 \beta \mu\right) \frac{\partial \beta}{\partial r}\right]
$$

where $I(r, \mu, \lambda)$ is the specific intensity scaled by $r^{2}, r$ is the radial coordinate, $\mu=\cos \theta$ is the cosine of the direction angle, $v$ is the velocity, $\beta=v / c, \gamma^{2}=$ $1 /\left(1-\beta^{2}\right), \chi(r, \lambda)$ is the total extinction coefficient, $\chi=\kappa+\sigma_{e}+\kappa_{l} \varphi(\lambda)$, and $\eta(r, \lambda)$ is the emissivity.

\subsection{Numerical solution}

The details of the numerical solution are discussed in detail in Hauschildt \& Baron (1999) so we only sketch the basic idea here. We discretize the $\partial / \partial \lambda$ term and treat the boundary value problem for each wavelength individually, using an Operator splitting (OS) method. The steps are: solve along characteristics of the RTE, using the piecewise parabolic ansatz to calculate $I$ for given $J$, and iterate to self-consistent solution for $J$ using a band diagonal accelerated lambda operator. The eigenvalues of amplification matrix are close to unity and the use of operator splitting reduces the eigenvalues of the amplification matrix.

\section{Statistical Equilibrium Equations}

The radiative transfer equation depends on the opacities and emissivity, which in turn depend on the level populations (which in turn depend on the radiation field). Thus, we must iterate the statistical equilibrium equations, simultaneously with the radiative transfer equation (the solution of the generalized radiative equilibrium condition is discussed in Hauschildt et al., 2003).

The rate equations are given by (see Mihalas \& Mihalas, 1984)

$$
\begin{aligned}
\sum_{j<i} n_{j}\left(R_{j i}+C_{j i}\right) & \\
- & n_{i}\left\{\sum_{j<i}\left(R_{i j}+C_{i j}\right)+\sum_{j>i}\left(\frac{n_{j}^{*}}{n_{i}^{*}}\right)\left(R_{i j}+C_{i j}\right)\right\} \\
& +\sum_{j>i} n_{j}\left(\frac{n_{j}^{*}}{n_{i}^{*}}\right)\left(R_{j i}+C_{j i}\right)=0 .
\end{aligned}
$$

\subsection{Solution of the Rate Equations}

Note that line and continuum scattering prevent the use of the $\Lambda$-iteration for the solution of the rate equations. Therefore, we use the Operator Splitting method (pre-conditioning) to define a "rate operator" in analogy to the $\Lambda$ operator (Hauschildt, 1993):

$$
R_{i j}=\left[R_{i j}\right][n] .
$$

We also define an "approximate rate operator" $\left[R_{i j}^{*}\right]$ and write the iteration scheme in the form:

$$
R_{i j}=\left[R_{i j}^{*}\right]\left[n_{\text {new }}\right]+\left(\left[R_{i j}\right]-\left[R_{i j}^{*}\right]\right)\left[n_{\text {old }}\right] .
$$


Inserting the above expression for $R_{i j}$ into the statistical equilibrium equations and linearizing we obtain,

$$
\begin{aligned}
& \sum_{j<i} n_{j, \text { old }}\left[R_{j i}^{*}\right]\left[n_{\text {new }}\right] \\
& -n_{i, \text { old }}\left\{\sum_{j<i}\left[R_{i j}^{*}\right]\left[n_{\text {new }}\right]+\sum_{j>i}\left(\frac{n_{j}^{*}}{n_{i}^{*}}\right)\left[R_{i j}^{*}\right]\left[n_{\text {new }}\right]\right\} \\
& +\sum_{j>i} n_{j, \text { old }}\left(\frac{n_{j}^{*}}{n_{i}^{*}}\right)\left[R_{j i}^{*}\right]\left[n_{\text {new }}\right] \\
& +\sum_{j<i} n_{j, \text { new }}\left(\left[\Delta R_{j i}\right]\left[n_{\text {old }}\right]+C_{j i}\right) \\
& -n_{i, \text { new }}\left\{\sum_{j<i}\left(\left[\Delta R_{i j}\right]\left[n_{\text {old }}\right]+C_{i j}\right)\right. \\
& \left.\quad+\sum_{j>i}\left(\frac{n_{j}^{*}}{n_{i}^{*}}\right)\left(\left[\Delta R_{i j}\right]\left[n_{\text {old }}\right]+C_{i j}\right)\right\} \\
& +\sum_{j>i} n_{j, \text { new }}\left(\frac{n_{j}^{*}}{n_{i}^{*}}\right)\left(\left[\Delta R_{j i}\right]\left[n_{\text {old }}\right]+C_{j i}\right)=0 .
\end{aligned}
$$

\section{The Computational Problem}

The size of the computational problem is determined by:

\section{- input data size}

- number of atomic/ionic spectral lines: $\approx 42 \times 10^{6} \rightarrow 0.6 \mathrm{~GB}$

- number of diatomic molecular lines: $\approx 35 \times 10^{6} \rightarrow 0.5 \mathrm{~GB}$

- number of hot water vapor lines:

* before 1994: $\approx 0.035 \times 10^{6}$

* 1994: $+\approx 6 \times 10^{6} \rightarrow 0.1 \mathrm{~GB}$

$* 1997:+\approx 330 \times 10^{6} \rightarrow 5 \mathrm{~GB}$

$* 2001:+\approx 100 \times 10^{6} \rightarrow 1.5 \mathrm{~GB}$

- total molecular lines (May 2001): $\approx 700 \times 10^{6} \rightarrow 10 \mathrm{~GB}$

All lines need to be accessed in a line selection procedure which dynamically creates sub-lists that can be as large as the original list, but are generally much smaller. This poses a significant data handling problem, which we have surmounted via the use of both memory and disk caches that allows us to trade (at run time) available memory for I/O bandwidth if required on a particular machine or architecture. 


\section{- memory/IO requirements}

- number of individual energy levels: $\approx 10,000 \rightarrow \approx 10 \mathrm{MB}$

- number of individual NLTE transitions: $\approx 100,000 \rightarrow \approx 150 \mathrm{MB}$

- EOS data storage $\approx 40 \mathrm{MB}$

- auxiliary storage $\approx 50 \mathrm{MB}$

- adds up to total memory requirement $\geq 200 \mathrm{MB}$

- number of individual energy levels and transitions has increased dramatically $\rightarrow$ memory requirements $>0.5 \mathrm{~GB}$ with the inclusion of more molecular species and the Chianti database.

\section{- (serial) CPU time}

- small for each individual point on the wavelength grid: $10 \ldots 100 \mathrm{msec}$

- number of wavelength points for radiative transfer: 30,000-500,000 (can be $>10^{6}$ ) $\rightarrow \approx 50,000$ sec to "sweep" once through all wavelength points

- typically $\approx 10$ iterations (sweeps) are required to obtain an equilibrium model $\rightarrow \approx 6$ CPU days

- there are, literally, 1000's of models in a typical grid ... The solution is parallel computing, which we have implemented in a MIMD model (Hauschildt et al., 1997a; Baron \& Hauschildt, 1998; Hauschildt et al., 2001; Baron et al., 2003). This dramatically reduces wallclock time per model and makes very large scale models feasible.

These numbers above are for models with 50 layers, with modern supercomputers many models use 100 or more layers and the scaling goes approximately as the cube of the number of layers.

\section{Applications}

We show a few illustrative applications of PHOENIX. Figure 1 displays the observed and synthetic spectrum of the Sun and the agreement is quite good. Figure 2 shows the observed and synthetic spectra of Vega, with excellent agreement in the UV and only a few features that are too strong in the optical compared to observations.

Turning from stars to supernovae, we note that supernovae are fundamentally different from stars, since in stars spectra only probe to the photosphere, whereas a time series of supernovae spectra allow us to "peel the onion" since geometrical dilution due to expansion of the ejecta causes the photosphere to receed in mass with time. Figure 3 shows the synthetic spectrum of the W7 deflagration model (Nomoto et al., 1984) compared to the observations of SN 1994D at 6 days prior to maximum light (Lentz et al., 2001a). Figure 4 shows that delayed detonation models well reproduce the spectra of SN 1984A - a "fast" SNe Ia (Lentz et al., 2001b; Hatano et al., 2000). 

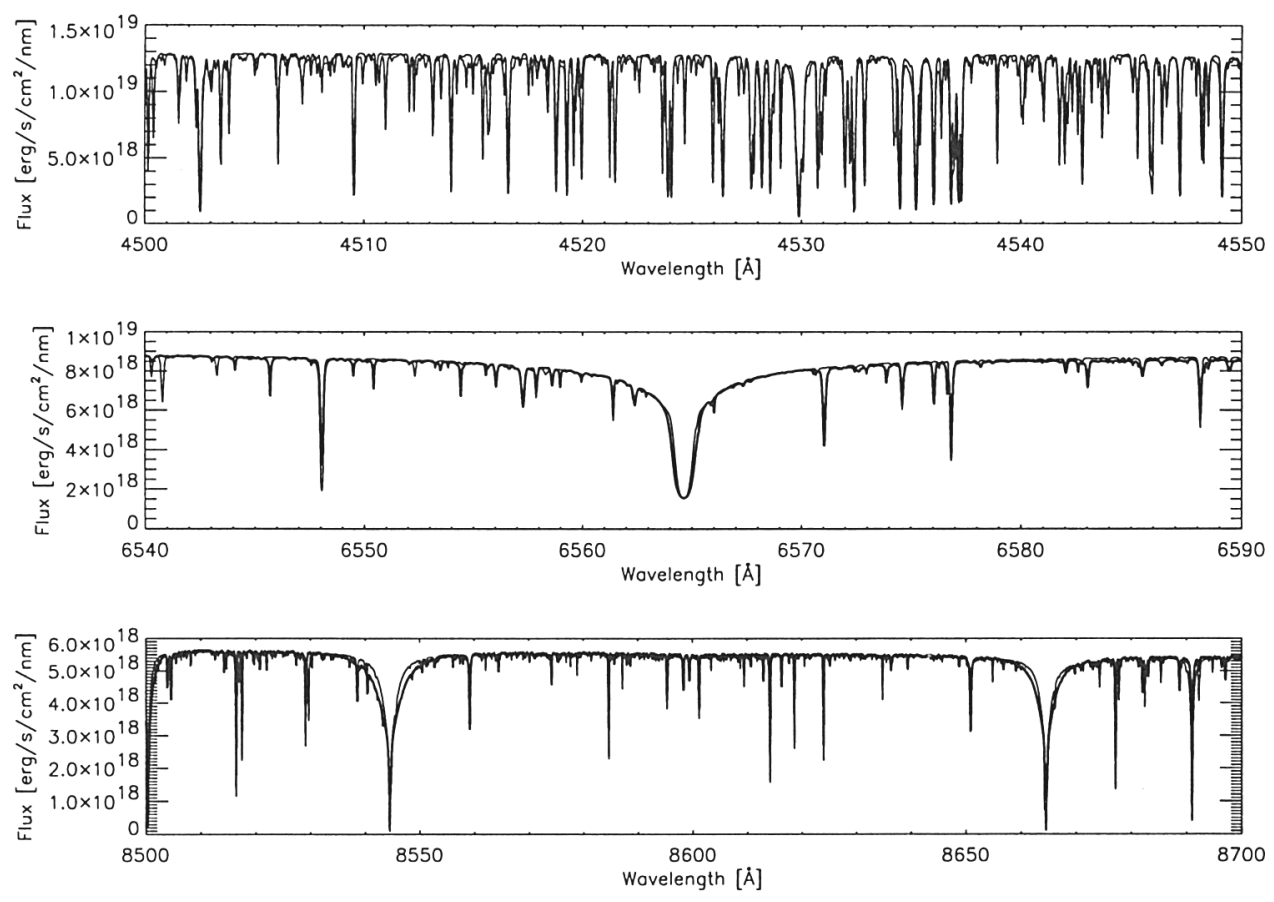

Figure 1. The synthetic model spectrum of the Sun (thin line) compared to observations.

We have also applied PHOENIX to core collapse supernovae. Figure 5 shows a very good fit to the first spectrum obtained of SN 1987A in both the UV and optical and Figure 6 shows the PHOENIX synthetic spectrum compared to SN 1987A at 4.5 days after the explosion. In order to reproduce $\mathrm{H} \alpha$, extra nickel mixing in the envelope was required. It is evident that the simple nickel mixing parameterization has destroyed the fit in the UV and clumping is probably required to fit the entire spectrum. Figure 7 shows that excellent fits to normal Type IIP spectra can be obtained at early times. These fits allow us to determine the reddening, amount of $\mathrm{H} / \mathrm{He}$ mixing, amount of nickel mixing, and the progenitor metallicity.

\section{Conclusions}

We have described the basic design of the generalized model atmosphere code PHOENIX. We have shown that good, but not perfect agreement with a variety of astrophysical objects can be obtained. Future work will include full time dependent calculations and full 3-D detailed NLTE radiative transfer. 


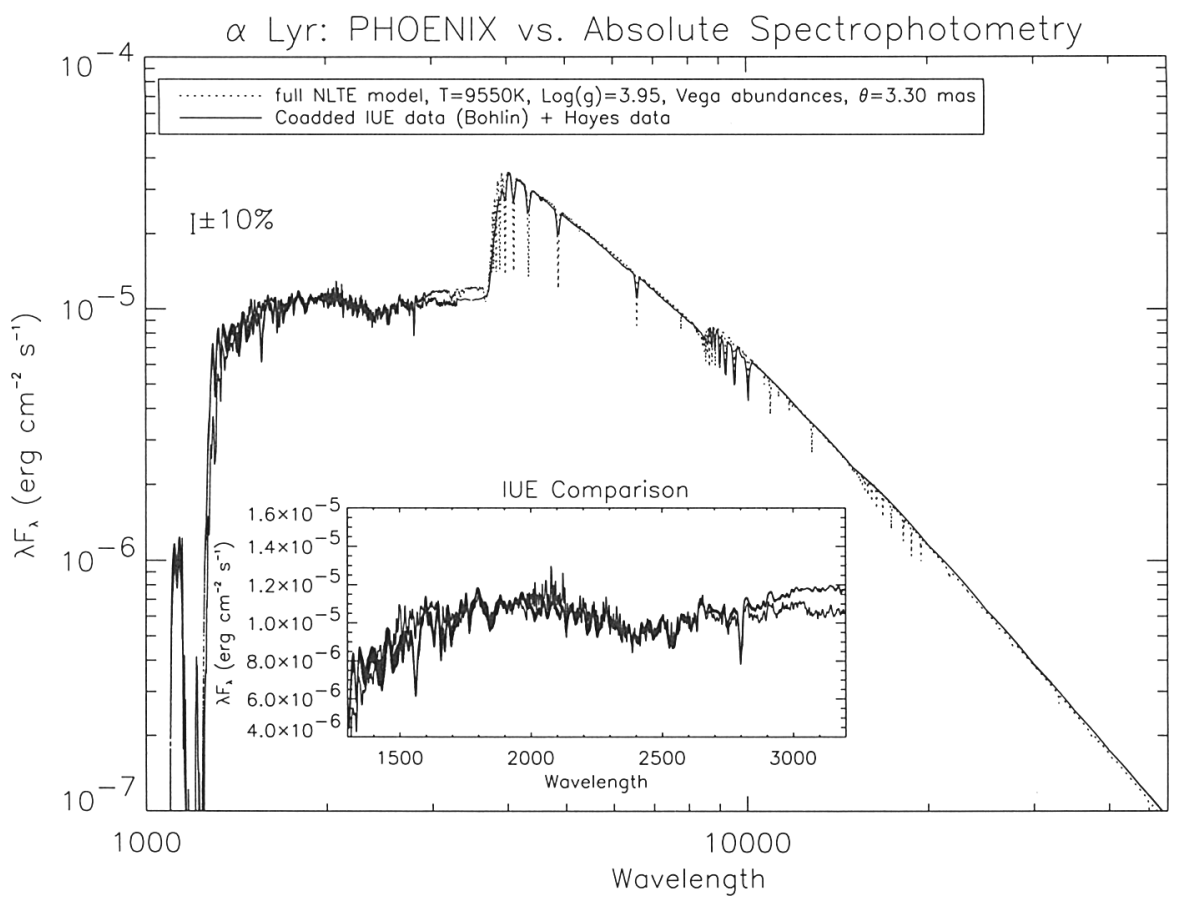

Figure 2. The synthetic model spectrum of Vega compared to observations (Hayes, 1985; Bohlin et al., 1990).

Acknowledgments. We thank our many collaborators who have contributed to the development of PHOENIX, in particular Ian Short, David Branch, Sumner Starrfield, and Steve Shore. This work was supported in part by NSF grant AST-9720704, NASA ATP grant NAG 5-8425 and LTSA grant NAG 5-3619 to the University of Georgia and by NASA grants NAG5-3505, NAG5-12127, NSF grant AST-0204771, and an IBM SUR grant to the University of Oklahoma. PHH was supported in part by the Pôle Scientifique de Modélisation Numérique at ENS-Lyon. Some of the calculations presented here were performed at the San Diego Supercomputer Center (SDSC), supported by the NSF, and at the National Energy Research Supercomputer Center (NERSC), supported by the U.S. DOE. We thank both these institutions for a generous allocation of computer time.

\section{Discussion}

KUBAT: In your calculation for high velocity fields (relativistic) you neglect the advection term in your rate equation. In addition, you assume radiative 


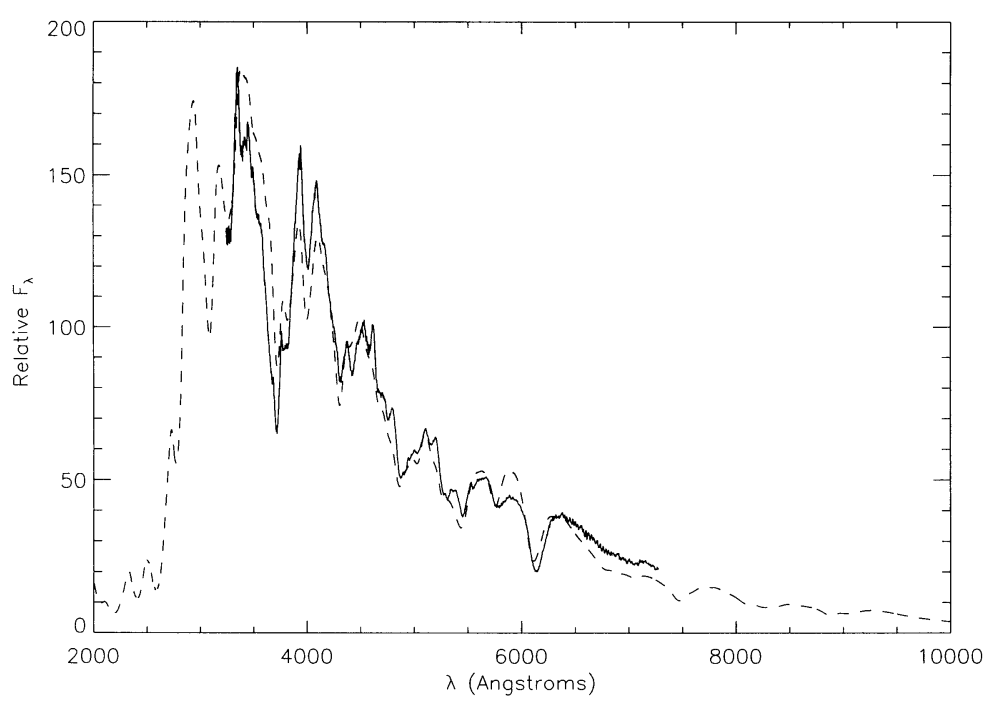

Figure 3. $\quad$ SN 1994D on 15 March 1994 (solid line, Filippenko, 1997) and W7 best fit synthetic spectrum for day 14 after explosion (dashed line), from Lentz et al. (2001a).

equilibrium. I do not think that these assumptions are acceptable for relativistic velocity fields. Can you explain your assumption?

BARON: We work in the co-moving frame, so advection does not enter into the rate equations, but is included in the transfer equations. We actually assume a generalized equation of radiative equilibrium i.e. total energy conservation so $\int K(J-B) d \lambda=S^{\prime} /(4 \pi)$ where $S^{\prime}$ is the rate of energy deposition from, e.g. mechanical heating or gamma ray deposition.

WOITKE: You said that you can handle non-LTE problems by using the same procedure for practically all astrophysical gases ranging from $100000 \mathrm{~K}$ to $100 \mathrm{~K}$. From my experience, collision rate are usually determined by experiments or by quantum mechanical calculations in very limited temperature ranges only. How do you treat the collision rates in PHOENIX?

BARON: We treat collisions using UR and semi-empirical formulae, except for some measured species like O I, Ne I, etc. But collisional rates are in fact the largest uncertainty in the atomic data.

ZAHN: How many parameters are adjusted to advise these excellent fits of SN spectra?

BARON: The parameters range from 1, the amount of gamma-ray deposition, given by a hydrodynamic explosion model to many, e.g. density exponent, metallicity, nickel mixing, hydrogen mixing, etc. However given a time series of spec- 


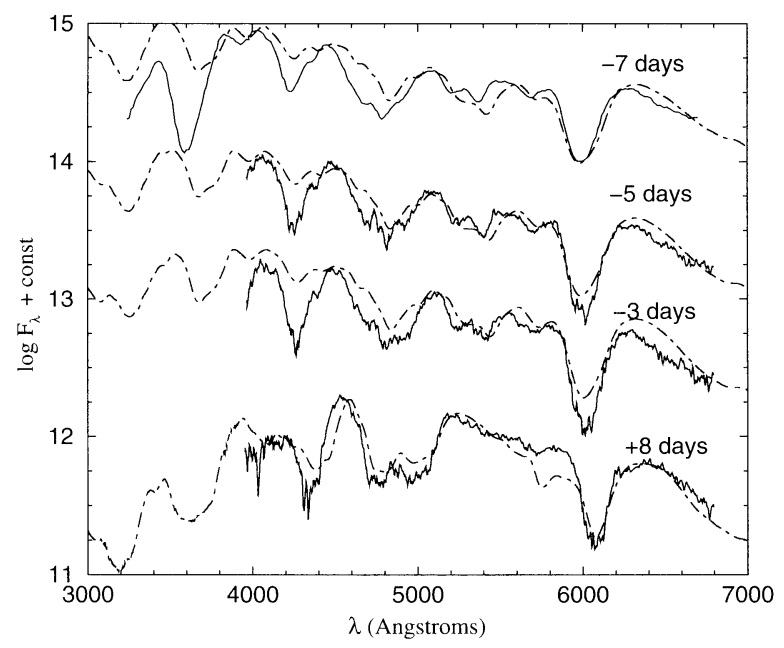

Figure 4. Synthetic spectra for the delayed detonation model 21c (dot-dashed lines) plotted against observed spectra for SN 1984A (solid lines) from Lentz et al. (2001b).

tra, the parameters can be determined to good accuracy (see Baron et al., which should be finished this summer). 


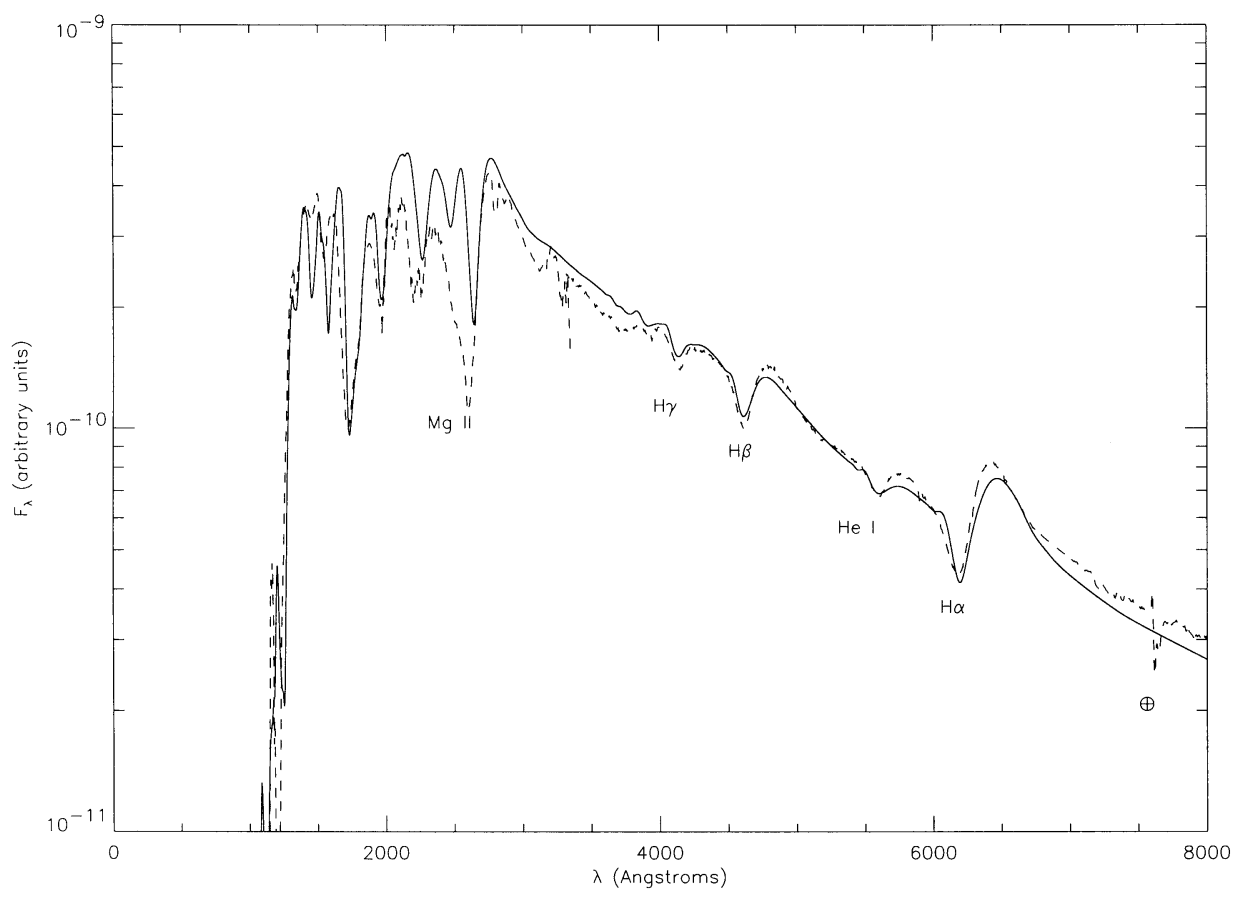

Figure 5. PHOENIX model spectrum (solid line) for day 1.36 (Mitchell et al., 2002). Important optical lines include $\mathrm{H} \alpha$ through $\mathrm{H} \delta$, and He I $\lambda 5876$. The optical spectra are taken from the CTIO archive (Phillips et al., 1988) and all UV spectra are from IUE (Pun et al., 1995). 


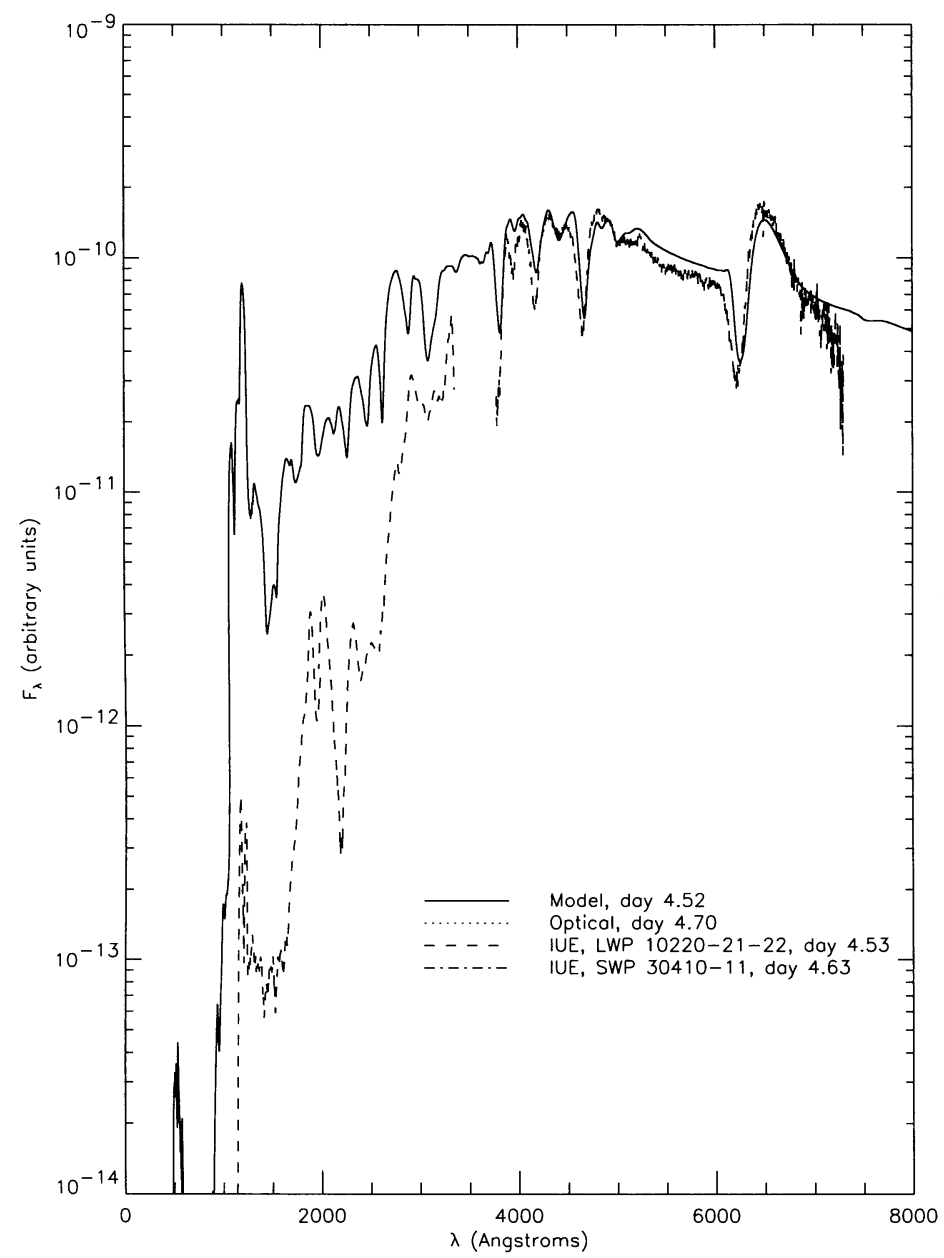

Figure 6. PHOENIX model spectrum for SN 1987A, day 4.52, with gamma-ray deposition calculated assuming local deposition due to a constant nickel mass fraction of $1.0 \times 10^{-3}$ everywhere in the envelope (Mitchell et al., 2002). 


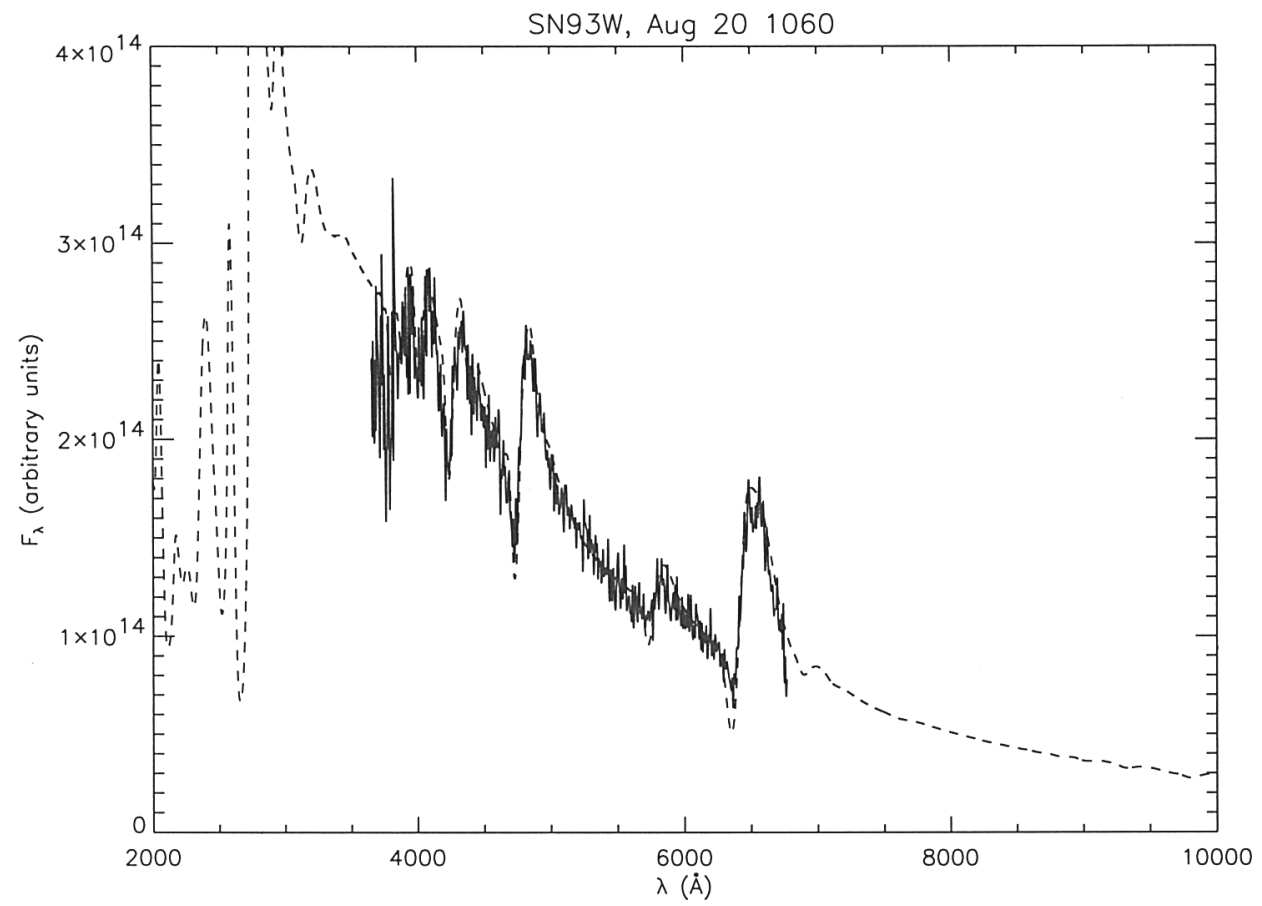

Figure 7. The observed spectrum of the Type IIP SN $1993 \mathrm{~W}$ compared to the synthetic spectrum. The agreement is excellent. 


\section{References}

Allard, F., Hauschildt, P. H., Alexander, D. R., Tamanai, A., \& Schweitzer, A. 2001, ApJ, 556, 357

Allen, C. W. 1973, Astrophysical Quantities, Third edn. (London: Athlone Press)

Aufdenberg, J. P., Hauschildt, P. H., \& Baron, E. 1999, MNRAS, 302, 599

Aufdenberg, J. P., Hauschildt, P. H., Baron, E., Nordgren, T. E., Burnley, A. W., Howarth, I. D., Gordon, K. D., \& Stansberry, J. A. 2002, ApJ, 570, 344

Aufdenberg, J. P., Hauschildt, P. H., Shore, S. N., \& Baron, E. 1998, ApJ, 498, 837

Barman, T. S., Hauschildt, P. H., Schweitzer, A., Stancil, P. C., Baron, E., \& Allard, F. 2002, ApJ, 569, L51

Baron, E., Branch, D., Hauschildt, P. H., Filippenko, A. V., \& Kirshner, R. P. 1999, ApJ, 527, 739

Baron, E. \& Hauschildt, P. H. 1998, ApJ, 495, 370

Baron, E., Hauschildt, P. H., \& Lowenthal, D. 2003, in Stellar Atmospheric Modeling, ed. I. Hubeny, D. Mihalas, \& K. Werner (San Francisco: ASP), in press

Baron, E., Hauschildt, P. H., \& Young, T. R. 1995, Physics Reports, 256, 23

Bohlin, R. C., Holm, A. V., Harris, A. W., \& Gry, C. 1990, ApJS, 73, 413

Filippenko, A. V. 1997, in Thermonuclear Supernovae, ed. P. Ruiz-Lapuente, R. Canal, \& J. Isern (Dordrecht: Kluwer), 1

Hatano, K., Branch, D., Lentz, E., Baron, E., Filippenko, A. V., \& Garnavich, P. 2000, ApJ, 543, L49

Hauschildt, P. H. 1992, JQSRT, 47, 433

—. 1993, JQSRT, 50, 301

Hauschildt, P. H., Allard, F., \& Baron, E. 1999, ApJ, 512, 377

Hauschildt, P. H., Allard, F., Ferguson, J., Baron, E., \& Alexander, D. 1999, ApJ, 525, 871

Hauschildt, P. H., Barman, T. S., Baron, E., \& Allard, F. 2003, in Stellar Atmospheric Modeling, ed. I. Hubeny, D. Mihalas, \& K. Werner (San Francisco: ASP), in press

Hauschildt, P. H. \& Baron, E. 1999, J. Comp. Applied Math., 109, 41

Hauschildt, P. H., Baron, E., \& Allard, F. 1997a, ApJ, 483, 390 
Hauschildt, P. H., Lowenthal, D. K., \& Baron, E. 2001, ApJS, 134, 323

Hauschildt, P. H., Schwarz, G., Baron, E., Starrfield, S., Shore, S., \& Allard, F. 1997b, ApJ, 490, 803

Hauschildt, P. H., Störzer, H., \& Baron, E. 1994, JQSRT, 51, 875

Hayes, D. S. 1985, in IAU Symp. 111: Calibration of Fundamental Stellar Quantities, Vol. 111, 225-249

Kurucz, R. 1993, CDROM No. 1: Atomic Data for Opacity Calculations, SAO Cambridge, MA

-. 1994a, CDROM No. 22: Atomic Data for Fe and Ni, SAO Cambridge, MA

-. 1994b, CDROM No. 23: Atomic Data for Mn and Co, SAO Cambridge, MA

Lentz, E., Baron, E., Branch, D., \& Hauschildt, P. H. 2001a, ApJ, 557, 266

-. 2001b, ApJ, 547, 402

Mathisen, R. 1984, Photo Cross Sections for Stellar Atmosphere Calculations, Tech. rep., Institute of Theoretical Astrophysics, Univ. of Oslo, unpublished

Mihalas, D. \& Mihalas, B. W. 1984, Foundations of Radiation Hydrodynamics (Oxford: Oxford University)

Mitchell, R., Baron, E., Branch, D., Hauschildt, P. H., Nugent, P., Lundqvist, P., Blinnikov, S., \& Pun, C. S. J. 2002, ApJ, 574, 293

Nomoto, K., Thielemann, F.-K., \& Yokoi, K. 1984, ApJ, 286, 644

Olson, G. L., Auer, L. H., \& Buchler, J. R. 1987, JQSRT, 38, 431

Olson, G. L. \& Kunasz, P. B. 1987, JQSRT, 38, 325

Phillips, M., Hamuy, M., Heathcote, S., Suntzeff, N., \& Kirhakos, S. 1988, AJ, 95,1087

Pun, C. S. J. et al. 1995, ApJS, 99, 223

Schwarz, G., Hauschildt, P. H., S. Starrfield, E. B., Allard, F., Shore, S., \& Sonneborn, G. 1997, MNRAS, 284, 669

Schweitzer, A., Gizis, J. E., Hauschildt, P. H., Allard, F., Howard, E. M., \& Kirkpatrick, J. D. 2002, ApJ, 566, 435

Schweitzer, A., Gizis, J. E., Hauschildt, P. H., Allard, F., \& Reid, N. 2001, ApJ, 555,368

Schweitzer, A., Hauschildt, P. H., \& Baron, E. 2000, ApJ, 541, 1004

Seaton, M. J., Yan, Y., Mihalas, D., \& Pradhan, A. K. 1994, MNRAS, 266, 805

Van Regemorter, H. 1962, ApJ, 136, 906

Verner, D. A. \& Yakovlev, D. G. 1995, A\&AS, 109, 125 